\title{
ON THE EXPONENT OF FINITE-DIMENSIONAL HOPF ALGEBRAS
}

\author{
Pavel Etingof and Shlomo Gelaki
}

\section{Introduction}

One of the classical notions of group theory is the notion of the exponent of a group. The exponent of a group is the least common multiple of orders of its elements.

In this paper we generalize the notion of exponent to Hopf algebras. We define the exponent of a Hopf algebra $H$ (with bijective antipode) to be the smallest $n$ such that $m_{n} \circ\left(I \otimes S^{-2} \otimes \cdots \otimes S^{-2 n+2}\right) \circ \Delta_{n}=\varepsilon \cdot 1$, where $m_{n}, \Delta_{n}, S, 1$, and $\varepsilon$ are the iterated product and coproduct, the antipode, the unit, and the counit. If $H$ is involutive (for example, $H$ is semisimple and cosemisimple), the last formula reduces to $m_{n} \circ \Delta_{n}=\varepsilon \cdot 1$.

We give four other equivalent definitions of the exponent (valid for finitedimensional Hopf algebras). In particular, we show that the exponent of $H$ equals the order of the Drinfeld element $u$ of the quantum double $D(H)$, and the order of $\mathcal{R}_{21} \mathcal{R}$, where $\mathcal{R}$ is the universal $R$-matrix of $D(H)$.

We show that the exponent is invariant under twisting. We prove that for semisimple and cosemisimple Hopf algebras $H$, the exponent is finite and divides $\operatorname{dim}(H)^{3}$. For triangular semisimple Hopf algebras in characteristic zero, we show that the exponent divides $\operatorname{dim}(H)$. These theorems are motivated by the work of Kashina [Ka1,Ka2], who conjectured that if $H$ is semisimple and cosemisimple then (using our language) the exponent of $H$ is always finite and divides $\operatorname{dim}(H)$, and showed that the order of the squared antipode of any finite-dimensional semisimple and cosemisimple Hopf algebra in the Yetter-Drinfeld category of $H$ divides the exponent of $H$.

At the end we formulate some open questions, in particular suggest a formulation for a possible Hopf algebraic analogue of Sylow's theorem.

\section{Definition and Elementary Properties of Exponent}

Let $H$ be a Hopf algebra over any field $k$, with multiplication map $m$, comultiplication map $\Delta$ and antipode $S$. We will always assume that $S$ is bijective. Let $m_{1}=I$ and $\Delta_{1}=I$ be the identity map $H \rightarrow H$, and for any integer $n \geq 2$ let $m_{n}: H^{\otimes n} \rightarrow H$ and $\Delta_{n}: H \rightarrow H^{\otimes n}$ be defined by $m_{n}=m \circ\left(m_{n-1} \otimes I\right)$, and $\Delta_{n}=\left(\Delta_{n-1} \otimes I\right) \circ \Delta$. We start by making the following definition.

Received December 28, 1998. 
Definition 2.1. The exponent of a Hopf algebra $H$, denoted by $\exp (H)$, is the smallest positive integer $n$ satisfying $m_{n} \circ\left(I \otimes S^{-2} \otimes \cdots \otimes S^{-2 n+2}\right) \circ \Delta_{n}=\varepsilon \cdot 1$. If such $n$ does not exist, we say that $\exp (H)=\infty$.

Let us list some of the elementary properties of $\exp (H)$.

Proposition 2.2. Let $H$ be a Hopf algebra over $k$. Then:

(1) The order of any group-like element of $H$ divides $\exp (H)$ (here we agree that any positive integer $n$ divides $\infty)$.

(2) For any group $G, \exp (k[G])$ equals the exponent of $G$ (see e.g. [Ro, p.12]), i.e. the least common multiple of the orders of the elements of $G$.

(3) If $\exp (H)=n<\infty$ then $m_{r} \circ\left(I \otimes S^{-2} \otimes \cdots \otimes S^{-2 r+2}\right) \circ \Delta_{r}=\varepsilon \cdot 1$ if and only if $r$ is divisible by $n$.

(4) If $H$ is finite-dimensional, $\exp \left(H^{*}\right)=\exp (H)$.

(5) $\exp \left(H_{1} \otimes H_{2}\right)$ equals the least common multiple of $\exp \left(H_{1}\right)$ and $\exp \left(H_{2}\right)$.

(6) If $\exp (H)=2$ then $H$ is commutative and cocommutative (this generalizes the fact that a group $G$ with $g^{2}=1$ for all $g \in G$ is abelian).

(7) The exponents of Hopf subalgebras and quotients of $H$ divide $\exp (H)$.

(8) If $K \supseteq k$ is a field then $\exp \left(H \otimes_{k} K\right)=\exp (H)$.

Proof. (1) Suppose $\exp (H)<\infty$, and set $n=\exp (H)$. Since $S^{2}(g)=g$ we have that $g^{n}=m_{n} \circ \Delta_{n}(g)=m_{n} \circ\left(I \otimes S^{-2} \otimes \cdots \otimes S^{-2 n+2}\right) \circ \Delta_{n}(g)=\varepsilon(g) 1=1$. Therefore the order of $g$ divides $n$.

(6) Since $m \circ\left(I \otimes S^{-2}\right) \circ \Delta=\varepsilon \cdot 1$ is equivalent to $S^{3}=I$, we have that $I: H \rightarrow H$ is an antiautomorphism of algebras and coalgebras, and the result follows.

The proofs of the other parts are obvious.

Remark 2.3. Part (2) of Proposition 2.2 motivated Definition 2.1.

Example 2.4. Let $H$ be a finite-dimensional Hopf algebra over an algebraically closed field $k$ of characteristic zero. Suppose that $H$ contains a non-trivial $1: g$ skew-primitive element $x$ (i.e. $\Delta(x)=x \otimes 1+g \otimes x$, where $g$ is a group-like element, and $x \notin k[g])$. It is clear that in this case we may assume that $x g=q g x$ for some root of unity $q$ of order dividing $|g|$. Also, $S^{2}(x)=q x, \varepsilon(x)=0$, $\left\{x, g x, \ldots, g^{|g|-1} x\right\}$ is linearly independent, and hence

$$
m_{n} \circ\left(I \otimes S^{-2} \otimes \cdots \otimes S^{-2 n+2}\right) \circ \Delta_{n}(x)=\sum_{i=0}^{n-1} q^{-i} g^{i} x \neq 0 .
$$

Hence, $\exp (H)=\infty$. In particular, the exponent of any pointed Hopf algebra $H$ over $k$ (which is not a group algebra) is $\infty$, since by [TW], $H$ contains a non-trivial skew-primitive element. 
In the sequel, we will assume for simplicity that $H$ is finite-dimensional. Let us formulate four equivalent definitions of $\exp (H)$. Recall that the Drinfeld double $D(H)=H^{* c o p} \otimes H$ of $H$ is a quasitriangular Hopf algebra with universal $R$-matrix $\mathcal{R}=\sum_{i} h_{i} \otimes h_{i}^{*}$, where $\left\{h_{i}\right\},\left\{h_{i}^{*}\right\}$ are dual bases for $H$ and $H^{*}$ respectively. Let $u=m(S \otimes I) \tau(\mathcal{R})=\sum_{i} S\left(h_{i}^{*}\right) h_{i}$, where $S$ is the antipode of $D(H)$ and $\tau$ is the usual flip map, be the Drinfeld element of $D(H)$. By [D],

$$
S^{2}(x)=u x u^{-1}, x \in D(H) \text { and } \Delta(u)=(u \otimes u)\left(\mathcal{R}_{21} \mathcal{R}\right)^{-1} .
$$

Theorem 2.5. Let $H$ be a finite-dimensional Hopf algebra over $k$. Then

(1) $\exp (H)$ equals the smallest positive integer $n$ such that

$$
\mathcal{R}\left(I \otimes S^{2}\right)(\mathcal{R}) \cdots\left(I \otimes S^{2 n-2}\right)(\mathcal{R})=1 .
$$

(2) $\exp (H)$ equals the order of $u$.

(3) $\exp (H)$ equals the order of $\mathcal{R}_{21} \mathcal{R}$.

(4) $\exp (H)$ equals the order of any non-zero element $v \in D(H)$ satisfying

$$
\Delta(v)=(v \otimes v)\left(\mathcal{R}_{21} \mathcal{R}\right)^{-1} .
$$

Proof. First note that since $(\Delta \otimes I)(\mathcal{R})=\mathcal{R}_{13} \mathcal{R}_{23}$, it follows that

$$
\left(\Delta_{n} \otimes I\right)(\mathcal{R})=\mathcal{R}_{1, n+1} \cdots \mathcal{R}_{n, n+1},
$$

for all $n$.

Second, recall that the map $H^{*} \otimes H \rightarrow D(H), p \otimes h \mapsto p h$ is a linear isomorphism $[\mathrm{D}]$.

Now we will show the equivalence of Definition 2.1 and the four definitions in the theorem.

(Definition $2.1 \Leftrightarrow 1 \Leftrightarrow 2$ ) Write $\mathcal{R}=\sum_{j} a_{j} \otimes b_{j}$. Using the above we obtain the following equivalences:

$$
\begin{array}{ll}
m_{n} \circ\left(I \otimes S^{-2} \otimes \cdots \otimes S^{-2 n+2}\right) \circ \Delta_{n}=\varepsilon \cdot 1 & \Longleftrightarrow \\
\left(m_{n} \circ\left(I \otimes S^{-2} \otimes \cdots \otimes S^{-2 n+2}\right) \circ \Delta_{n} \otimes I\right)(\mathcal{R})=1 & \Longleftrightarrow \\
\left(m_{n} \circ\left(I \otimes S^{-2} \otimes \cdots \otimes S^{-2 n+2}\right) \otimes I\right)\left(\mathcal{R}_{1, n+1} \cdots \mathcal{R}_{n, n+1}\right)=1 & \Longleftrightarrow \\
\sum_{i_{1}, \ldots, i_{n}} a_{i_{1}} S^{-2}\left(a_{i_{2}}\right) \cdots S^{-2 n+2}\left(a_{i_{n}}\right) \otimes b_{i_{1}} \cdots b_{i_{n}}=1 & \Longleftrightarrow \\
\mathcal{R}\left(I \otimes S^{2}\right)(\mathcal{R}) \cdots\left(I \otimes S^{2 n-2}\right)(\mathcal{R})=1 & \Longleftrightarrow \\
u^{n}=1 &
\end{array}
$$

(in the last step we applied $m \circ(I \otimes S) \tau$ to both sides of the equation, and used the fact that $u S^{-2}(x)=x u$, for all $\left.x \in D(H)\right)$.

$(2 \Leftrightarrow 3)$ Clearly if $u^{n}=1$ then $\left(\mathcal{R}_{21} \mathcal{R}\right)^{n}=1$. In the other direction, first note that $\left(\mathcal{R}_{21} \mathcal{R}\right)^{n}=1$ implies that $u^{n} \in G(D(H)$ ) (where $G(A)$ is the group of 
grouplike elements of a Hopf algebra $A$ ). Therefore by $[\mathrm{R}], u^{n}=a b$ where $a \in G\left(H^{*}\right)$ and $b \in G(H)$. Regarding $u$ as an element of $H^{*} \otimes H$, we have that $m(I \otimes \varepsilon)(u)=m(\varepsilon \otimes I)(u)=1$. Hence it follows that $1=m(I \otimes \varepsilon)\left(u^{n}\right)=a$ and $1=m(\varepsilon \otimes I)\left(u^{n}\right)=b$, so $u^{n}=1$.

$(2 \Leftrightarrow 4)$ First note that $v=u g$, where $g \in G(D(H))$. Since $g$ commutes with $u$ we have that $v^{n}=u^{n} g^{n}$. Therefore if $u^{n}=1$ then $v^{n}=1$ by parts 1 and 3 of Proposition 2.2, and if $v^{n}=1$ then $u^{n} \in G(D(H))$, so $u^{n}=1$ as explained above.

Corollary 2.6. Let $H$ be a finite-dimensional Hopf algebra over $k$. Then

$$
\exp \left(H^{c o p}\right)=\exp \left(H^{o p}\right)=\exp (H) .
$$

Proof. Since $\left(D\left(H^{* c o p}\right), \tilde{\mathcal{R}}\right) \cong\left(D(H)^{o p}, \mathcal{R}_{21}\right)$ as quasitriangular Hopf algebras, it follows from part 1 of Theorem 2.5 that $\exp \left(H^{* c o p}\right)=\exp (H)$. Hence the result follows from part 4 of Proposition 2.2.

\section{Invariance of Exponent Under Twisting}

In this section we show that $\exp (H)$ is invariant under twisting. First recall Drinfeld's notion of a twist for Hopf algebras.

Definition 3.1. Let $H$ be a Hopf algebra over $k$. A twist for $H$ is an invertible element $J \in H \otimes H$ which satisfies

$$
(\Delta \otimes I)(J) J_{12}=(I \otimes \Delta)(J) J_{23} \text { and }(\varepsilon \otimes I)(J)=(I \otimes \varepsilon)(J)=1 .
$$

Given a twist $J$ for $H$, we can construct a new Hopf algebra $H^{J}$, which is the same as $H$ as an algebra, with coproduct $\Delta^{J}$ given by

$$
\Delta^{J}(x)=J^{-1} \Delta(x) J, x \in H .
$$

If $(H, R)$ is quasitriangular then so is $H^{J}$ with the $R$-matrix

$$
R^{J}=J_{21}^{-1} R J
$$

In particular, since $H$ is a Hopf subalgebra of $D(H)$, we can twist $D(H)$ using the twist $J \in D(H) \otimes D(H)$ and obtain $\left(D(H)^{J}, \mathcal{R}^{J}\right)$.

Proposition 3.2. Let $H$ be a finite-dimensional Hopf algebra over $k$, and let $J$ be a twist for $H$. Then $\left(D\left(H^{J}\right), \mathcal{R}\right) \cong\left(D(H)^{J}, \mathcal{R}^{J}\right)$ as quasitriangular Hopf algebras.

Proof. Let $H_{+}$and $H_{-}$be the Hopf subalgebras of $D(H)^{J}$ generated by the left and right components of $\mathcal{R}^{J}$ respectively. Clearly, $H_{+} \subseteq H^{J}$. In order to prove the theorem it is sufficient to prove that the multiplication map $H_{+} \otimes H_{-} \rightarrow$ $D(H)^{J}$ is a linear isomorphism, since then $H_{+}=H^{J}$ (by dimension counting) and the result will follow.

Clearly, $\operatorname{dim}\left(H_{+}\right) \leq \operatorname{dim}(H)$ and $\operatorname{dim}\left(H_{-}\right)=\operatorname{dim}\left(H_{+}\right)$, so we need to show that $H_{+} H_{-}=D(H)$. Since $J \mathcal{R}_{21}^{J} \mathcal{R}^{J} J^{-1}=\mathcal{R}_{21} \mathcal{R}$ we have that $H H_{-} H=$ 
$D(H)$ (looking at the first component). Let $A=H_{+} H_{-}=H_{-} H_{+}, \operatorname{dim}(H)=$ $d, \operatorname{dim}\left(H_{+}\right)=d_{+},\left\{v_{1}, \ldots, v_{d / d_{+}}\right\}$with $v_{1}=1$ be a basis of $H$ as a right $H_{+}$-module, and $\left\{w_{1}, \ldots, w_{d / d_{+}}\right\}$with $w_{1}=1$ be a basis of $H$ as a left $H_{+}$-module (such bases exist by the freeness theorem for Hopf algebras [NZ]). Then we get by dimension counting that $D(H)=\bigoplus_{i, j=1}^{d / d_{+}} v_{i} A w_{j}$. Thus, $H H_{-} \cap$ $H_{-} H=A$, hence $H \subseteq A$ which implies that $H A H=A$, and the result follows.

Theorem 3.3. Let $H$ be a finite-dimensional Hopf algebra over $k$, and let $J$ be a twist for $H$. Then $\exp (H)=\exp \left(H^{J}\right)$.

Proof. By part 3 of Theorem 2.5, and Proposition 3.2, it is sufficient to show that the order of $\mathcal{R}_{21}^{J} \mathcal{R}^{J}$ equals to the order of $\mathcal{R}_{21} \mathcal{R}$. But this is clear since they are conjugate.

Corollary 3.4. Let $H$ be a finite-dimensional Hopf algebra over $k$. Then $\exp (D(H))=\exp (H)$.

Proof. By [RS], there exists $J \in D(H) \otimes D(H)$ such that

$$
D(D(H)) \cong(D(H) \otimes D(H))^{J}
$$

as Hopf algebras. Then using Theorem 3.3 we get that $\exp (D(H))$ equals the order of $u$ in $(D(H) \otimes D(H))^{J}$ which equals the order of $u$ in $D(H) \otimes D(H)$, and hence equals $\exp (H)$ ( since $\left.u_{D(H) \otimes D(H)}=u_{D(H)} \otimes u_{D(H)}\right)$.

\section{The Exponent of a Semisimple and Cosemisimple Hopf Algebra}

In this section, we will show that if $H$ is semisimple and cosemisimple then $\exp (H)$ is finite, and give an estimate for it in terms of $\operatorname{dim}(H)$.

Let $H$ be a semisimple and cosemisimple Hopf algebra over $k$ (note that by $[\mathrm{LR}]$ the cosemisimplicity assumption is redundant if the characteristic of $k$ is $0)$. Recall that for semisimple and cosemisimple $H, D(H)$ is also semisimple and cosemisimple [R]. Also, by [LR, EG2], $S^{2}=I$ and hence $u$ is central in $D(H)$. This implies that $\exp (H)$ equals the smallest positive integer $n$ satisfying $m_{n} \circ \Delta_{n}=\varepsilon \cdot 1$, and also to the order of $\mathcal{R}$ (by part 1 of Theorem 2.5).

Remark 4.1. In [Ka1, Ka2] Kashina studied the smallest positive integer $n$ satisfying $m_{n} \circ \Delta_{n}=\varepsilon \cdot 1$, for any finite-dimensional Hopf algebra $H$. In particular she observed the analogous properties listed in Proposition 2.2, and proved an analogue to Corollary 3.4 under the assumption that this smallest $n$ is the same for $H$ and $H^{c o p}$.

Theorem 4.2. Let $(H, R)$ be a semisimple triangular Hopf algebra over a field $k$ of characteristic 0 . Then $\exp (H)$ divides $\operatorname{dim}(H)$. 
Proof. By part 8 of Proposition 2.2, we may assume that $k$ is algebraically closed. Now, it is straightforward to check that the theorem holds for $(k[G], 1 \otimes 1)$ where $G$ is a finite group. But by [EG1, Theorem 2.1], there exist a finite group $G$ and a twist $J \in k[G] \otimes k[G]$ such that $H \cong k[G]^{J}$ as Hopf algebras. Hence the result follows from Theorem 3.3.

Theorem 4.3. Let $H$ be a semisimple and cosemisimple Hopf algebra over $k$. Then $\exp (H)$ divides $\operatorname{dim}(H)^{3}$.

Theorem 4.3 will be proved later.

Corollary 4.4. Let $H$ be a semisimple and cosemisimple Hopf algebra over $k$, and let $B$ be a finite-dimensional semisimple Hopf algebra in the category of Yetter-Drinfeld modules over $H$. Then the order of the antipode of $B$ is finite and divides $2 \operatorname{dim}(H)^{3}$, and if $H$ is semisimple triangular and the characteristic of $k$ is 0 , then the order of the antipode of $B$ is finite and divides $2 \operatorname{dim}(H)$.

Proof. Follows from Theorems 4.2 and 4.3, and [Ka1, Theorem 6].

Remark 4.5. Theorem 4.3 is motivated by Vafa's theorem [V]. Vafa's theorem (see [Ki] for the mathematical exposition) states that the twists in a semisimple modular category act on the irreducible objects by multiplication by roots of unity. Thus, the fact that $u \in D(H)$ has a finite order follows from the fact that the category of representations of $D(H)$ is modular, with system of twists given by the action of the central element $u$ (see e.g. [EG1]).

Kashina conjectured the following:

Conjecture 4.6. Let $H$ be a semisimple and cosemisimple Hopf algebra over $k$. Then $\exp (H)$ is finite and divides $\operatorname{dim}(H)$.

This conjecture was checked by Kashina in a number of special cases [Ka1, Ka2]. Our results presented above give a proof to the first part of the conjecture, and additional supportive evidence for its second part.

Now we will prove Theorem 4.3. In order to do this, we need a lemma.

Lemma 4.7. Let $H$ be a Hopf algebra of finite dimension $d$ over $k, \mathcal{R} \in H \otimes$ $H^{* \text { cop }} \subset D(H) \otimes D(H)$ be the universal $R$-matrix, and $u \in D(H)$ be the Drinfeld element. Then:

(1) For any finite-dimensional $H$-module $V_{+}$and finite-dimensional $H^{*}$-module $V_{-}$, one has $\left(\operatorname{det}\left(\mathcal{R}_{\mid V_{+} \otimes V_{-}}\right)\right)^{d}=1$.

(2) For any finite-dimensional $D(H)$-module $V$, one has $\left(\operatorname{det}\left(u_{\mid V}\right)\right)^{d^{2}}=1$. 
Proof. (1) Recall that $(\Delta \otimes I)(\mathcal{R})=\mathcal{R}_{13} \mathcal{R}_{23}$. Apply this identity to $V_{+} \otimes H \otimes V_{-}$, where $H$ is the regular representation of $H$. Since $V_{+} \otimes H=\left(\operatorname{dim} V_{+}\right) H$, this yields, after taking determinants:

$$
\left(\operatorname{det}\left(\mathcal{R}_{\mid H \otimes V_{-}}\right)\right)^{\operatorname{dim} V_{+}}=\left(\operatorname{det}\left(\mathcal{R}_{\mid V_{+} \otimes V_{-}}\right)\right)^{d}\left(\operatorname{det}\left(\mathcal{R}_{\mid H \otimes V_{-}}\right)\right)^{\operatorname{dim} V_{+}} .
$$

The result follows after cancellation.

(2) We use Drinfeld's formula, $\Delta(u)=(u \otimes u)\left(\mathcal{R}_{21} \mathcal{R}\right)^{-1}$. Using part 1, and the fact that $D(H)=H^{*} \otimes H$ as $H^{*}$-module and $H$-module, we compute:

$$
\operatorname{det}\left(\Delta(u)_{\mid V \otimes D(H)}\right)=\left(\operatorname{det}\left(u_{\mid V}\right)\right)^{d^{2}}\left(\operatorname{det}\left(u_{\mid D(H)}\right)\right)^{\operatorname{dim} V} .
$$

Since $V \otimes D(H)=(\operatorname{dim} V) D(H)$, the result follows.

Proof of Theorem 4.3. By part 8 of Proposition 2.2, we may assume that $k$ is algebraically closed. Since $u$ is central, we have for any irreducible $D(H)$-module $V$ that $\operatorname{det}\left(u_{\mid V}\right)=\lambda(u, V)^{\operatorname{dim} V}$, where $\lambda(u, V)$ is the eigenvalue of $u$ on $V$. So by Lemma $4.7, \lambda(u, V)^{\operatorname{dim} V \cdot d^{2}}=1$. But by [EG1, Theorem 1.5], and in positive characteristic by [EG2, Theorem 3.7], $\operatorname{dim} V$ divides $d$, so $\lambda(u, V)^{d^{3}}=1$. Thus, $u^{d^{3}}=1$ and we are done by part 2 of Theorem 2.5 .

In the non-semisimple case, as we know, Theorem 4.3 fails, and the order of $u$ may be infinite. The analogue of Theorem 4.3 in this case is the following theorem.

Let $A$ be a finite-dimensional algebra. For any two irreducible $A$-modules $V_{1}$ and $V_{2}$, write $V_{1} \sim V_{2}$ if they occur as constituents in the same indecomposable $A$-module. Extend $\sim$ to an equivalence relation. For an irreducible module $W$, let $[W]$ be the equivalence class of $W$. For an indecomposable module $V$ let $[V]$ be the equivalence class of any constituent $W$ of $V$. Let $N_{V}$ be the greatest common divisor of dimensions of elements of $[\mathrm{V}]$.

Theorem 4.8. Let $H$ be a Hopf algebra of dimension $d$ over an algebraically closed field $k$. Then:

(1) For any indecomposable $D(H)$-module $V$, the unique eigenvalue of the central element $z=u S(u)$ on $V$ is a root of unity of order dividing $d^{2} N_{V}$.

(2) For any indecomposable $D(H)$ - module $V$, every eigenvalue of $u$ on $V$ is a root of unity of order dividing $2 d^{2} N_{V}$ (so the eigenvalues of $u$ on any $D(H)$ module are roots of unity).

Proof. (1) Recall that $z=u^{2} g$, where $g$ is a grouplike element of $D(H)$. By [NZ], the order of $g$ divides $\operatorname{dim}(H)=d$. Since $V$ is indecomposable and $z$ is central, $z$ has a unique eigenvalue $\lambda(z, V)$ on $V$. For any $W \in[V], \lambda(z, V)=\lambda(z, W)$, so we get, $\left(\operatorname{det}\left(u_{\mid W}\right)\right)^{2 d}=\lambda(z, V)^{d \cdot \operatorname{dim} W}$, which implies by part 2 of Lemma 4.7, that $\lambda(z, V)^{d^{2} \cdot \operatorname{dim} W}=1$. 
(2) Since, any eigenvalue $\mu$ of $u_{\mid V}$ has the property $\mu^{2}=\lambda(z, V) \nu$, where $\nu$ is an eigenvalue of $g^{-1}$, we have by part 1 that $\mu^{2 d^{2} \operatorname{dim} W}=1$, and the result follows.

Corollary 4.9. If $\exp (H)=\infty$, then $u$ is not semisimple.

Corollary 4.10. Let $H$ be a finite-dimensional Hopf algebra over a field $k$ of positive characteristic $p$. Then $\exp (H)<\infty$.

Proof. Let $u$ be the Drinfeld element of $D(H)$. By part 2 of Theorem 4.8, the eigenvalues of $u$ are roots of unity. Hence there exists a positive integer $a$ such that $u^{a}=1+n$ where $n \in D(H)$ is a nilpotent element. But then $u^{a p^{b}}=1$ for a sufficiently large positive integer $b$, and the result follows from part 2 of Theorem 2.5.

\section{Concluding Remarks}

In conclusion we would like to formulate some questions.

Question 5.1. Suppose that $H$ is a semisimple and cosemisimple Hopf algebra of dimension d over $k$. If a prime $p$ divides $d$, must it divide $\exp (H)$ ?

We do not know the answer to this question even in characteristic zero, even for $p=2$. However, if $H$ is a group algebra then the answer is positive, since the statement is equivalent to (a special case of) Sylow's first theorem: a finite group whose order is divisible by $p$ has an element of order $p$. So positive answer to Question 5.1 would be a "quantum Sylow theorem".

Question 5.2. Let $H$ be a semisimple and cosemisimple Hopf algebra over $k$ whose exponent is a power of a prime $p$. Must the dimension of $H$ be a power of the same prime?

This is a special case of Question 5.1, but we still do not know the answer, except for the case $\exp (H)=2$, when the answer is trivially positive. For group algebras, the statement is equivalent to the well-known group-theoretical result that a finite group where orders of all elements are powers of $p$ is a p-group (a special case of Sylow's theorem).

Question 5.3. Let $H$ be a finite-dimensional Hopf algebra over $k$ such that the element $u \in D(H)$ is semisimple in the regular representation. Does it follow that $H$ and $H^{*}$ are semisimple

(1) In characteristic zero?

(2) In positive characteristic $p$ ? 
By Theorem 4.8, part (1) of Question 5.3 is equivalent to the question whether for a finite-dimensional Hopf algebra $H$ in characteristic 0 , $\exp (H)<\infty$ implies that $H$ is semisimple.

A positive answer to part (2) of Question 5.3 implies a positive answer to Question 5.1 for involutive Hopf algebras defined over $\mathbb{Z}$ and free as $\mathbb{Z}$-modules (which includes group algebras, i.e. this would generalize Sylow's theorem). Indeed, if $H$ is such a Hopf algebra then for any prime $p$ dividing the dimension of $H$, either $H / p H$ or $(H / p H)^{*}$ is not semisimple (as $\operatorname{tr}\left(S^{2}\right)=0$ ), and hence $D(H)$ is not semisimple. If the answer to part (2) of Question 5.3 is positive, then this would imply that $u$ is not semisimple over $F_{p}$, i.e. the order of $u$ is divisible by $p$, as desired.

For group algebras, the answer to part (2) of Question 5.3 is positive: in this case semisimplicity of $u$ is equivalent to semisimplicity of $R=\sum g \otimes \delta_{g}$, which implies that all group elements $g$ are semisimple. This would imply that their orders are not divisible by $p$, which by Sylow's theorem implies that $p$ does not divide the order of the group.

\section{Acknowledgment}

The authors thank Yevgenia Kashina for suggesting the problem, and Susan Montgomery for useful discussions.

\section{References}

[D] V. Drinfeld, Almost cocommutative Hopf algebras, Leningrad Math. J. 1 (1990), 321342.

[EG1] P. Etingof and S. Gelaki, Some properties of finite-dimensional semisimple Hopf algebras, Math. Res. Lett. 5 (1998), 191-197.

[EG2] P. Etingof and S. Gelaki, On finite-dimensional semisimple and cosemisimple Hopf algebras in positive characteristic, Internat. Math. Res. Notices 16 (1998), 851-864.

[Ka1] Y. Kashina, On the order of the antipode of Hopf algebras in ${ }_{H}^{H} \mathcal{Y} D$, Comm. Algebra 27 (1999), 1261-1273.

[Ka2] Y. Kashina, A generalized power map for Hopf algebras, Hopf Algebras and Quantum Groups, Proceedings of the 1998 Brussels Conference, (S. Caenpeel, ed.), Marcel Dekker, to appear.

[Ki] A.A. Kirillov, Jr., On an inner product in modular tensor categories, J. Amer. Math. Soc. 9 (1996), 1135-1169.

[LR] R.G. Larson and D.E. Radford, Finite-dimensional cosemisimple Hopf algebras in characteristic 0 are semisimple, J. Algebra 117 (1988), 267-289.

[NZ] W.D. Nichols and M.B. Zoeller, A Hopf algebra freeness theorem, Amer. J. Math. 111 (1989), 381-385.

[R] D.E. Radford, Minimal quasitriangular Hopf algebras, J. Algebra 157 (1993), 285315.

[RS] N. Reshetikhin and M. Semenov-Tian-Shansky, Quantum R-matrices and factorization problems, J. Geom. Phys. 4 (1988), 533-550.

[Ro] D.J.S. Robinson, A course in the theory of groups, Second edition. Graduate Texts in Mathematics, 80., Springer-Verlag, New York, (1996).

[TW] E.J. Taft and R.L. Wilson, On antipodes in pointed Hopf algebras, J. Algebra 29 (1974), 27-32. 
[V] C. Vafa, Towards classification of conformal theories, Phys. Lett. B 206 (1988), 421426.

Department of Mathematics, Harvard University, Cambridge, MA 02138

E-mail address: etingof@abel.math.harvard.edu

Department of Mathematics, University of Southern California, los Angeles, CA 90089

E-mail address: gelaki@mathg.usc.edu 